\title{
Ventricular Trigeminy by ECG Finding
}

National Cancer Institute

\section{Source}

National Cancer Institute. Ventricular Trigeminy by ECG Finding. NCI Thesaurus. Code C71055.

An electrocardiographic finding of two sinus beats followed by a premature ventricular complex for 3 or more consecutive cycles; a regularly irregular rhythm of normal and abnormal QRS complexes in a 2-1 ratio. (CDISC) 